\title{
Multidisciplinary treatment of pain caused by bone metastases in cancer patients: two case reports
}

\author{
Natalia Cichowska-Cwalińska, Renata Zaucha
}

Over $50 \%$ patients with malignant neoplasms suffer pain. In Poland, about 200,000 people annually have to contend with this problem. It can arise from the prolonged lifespan of cancer patients, the development of iatrogenic pain syndromes, pain associated with cancer cachexia, or decreased efficacy of analgesics due to induced opioid tolerance. The commonly used term "cancer pain" actually refers to all types of pain. In order that each patient is optimally treated, it is necessary to identify the type and cause of their pain. One of the more difficult types of pain to treat is that due to bone metastases; with its inflammatory and neuropathic components. Every patient has the right to proper pain treatment, with the goal of achieving the maximum analgesic effect while minimizing any side effects.

NOWOTWORY J Oncol 2017; 67, 2: 142-145

Key words: cancer pain, neuropathic pain, bone metastasis, breakthrough pain

\section{Introduction}

Pain in cancer patients is a very complex phenomenon. It is primarily the result of proliferative disease, but also due to intense and sometimes aggressive oncological treatment. This is why about $30 \%$ of recovered patients experience such pain [1]. Cancer patients are living increasingly longer and are more likely to develop iatrogenic pain syndromes, cachexia-induced pain or decreased efficacy of analgesics due to opioid tolerance. In order to establish optimal treatments for each patient, it is necessary to identify the type and cause of their pain. According to the neurophysiological classification, pain can be distinguished into pain due to receptors (conjunctive pain) or neuropathic pain. Both are caused by mechanical or chemical sensitization of sensory receptors, with the difference that nociceptive pain arises in a properly functioning system of detection, conduction and modulation of pain whilst neuropathic pain shows evidence of damage to the nervous system, often by tumors or damaged bone structures. Conjunctive pain usually responds well to non-opioid analgesics and opioids as opposed to neuropathic pain which is resistant to analgesics, frequently requiring additional medication or other methods. Various tools are used to assess pain intensity. The easiest for patients and therefore the most commonly used tool is the numerical rating score (NRS), which consists of 11 degrees of pain intensity ranging from 0 to 10 , where 0 signifies the total absence of pain, and 10 is the worst possible pain.

One of the more difficult types of pain to treat is pain due to bone metastasis, with its inflammatory and neuropathic components. It is the most common pain with a dominant somatic mechanism, which is the result of activation of the sensory and sympathetic innervation of the periosteum, bone tissue and bone marrow by a series of substances released by the tumor and immune cells. This also becomes exacerbated by other disorders such as osteolysis of the bones, which irritates nociceptors that respond to a drop in $\mathrm{pH}$, or periosteal extension in the case of unstable fractures [2]. Sometimes, nerve growth factor (NGF) is released by the tumor cells, which results in nerve fiber growth in the bone itself, resulting in a neuropathic component that is very difficult to treat [2].

\section{Case no 1}

A 61-year-old patient was admitted to the accident and emergency (A\&E) department of a Gdańsk hospital due to an episode of very severe lumbar spine pain, in July 
2015. This pain had been treated for several months with non-steroidal anti-inflammatory analgesics. She received as needed, adjuvant ketoprofen $100 \mathrm{mg}$ up to 5 times daily. The patient was additionally treated for hypertension which was well controlled by taking enalapril 10 mg daily. In 2003, she had resection of IV and V segments of the left lung due to lung cancer (adenocarcinoma, G1, pT1aNOM0). In 2004, she then had a hysterectomy because of uterine fibroids. She has never smoked any tobacco products.

Magnetic resonance imaging (MR) showed a proliferative lesion of the L4 vertebral body, which could correspond to a primary or secondary osteosclerotic lesion. This could be a primary process or a secondary osteosclerotic change. In August 2015, a resection of the L4 pathological mass was performed at the Department of Neurosurgery, with simultaneous cementoplasty and transpedicular stabilization of L3-L5 vertebral bodies. Histopathological results clearly defined the nature of the lesion as a metastatic adenocarcinoma with the most likely exit point being from lung tissue (TTF1+). Diagnostics included positron emission tomography (PET/CT) which showed the presence of a metabolically active proliferative process in the upper lobe of the left lung, measuring $33 \mathrm{~mm} \times 19 \mathrm{~mm}$ (SUV up to 9.8) with dissemination to the skeletal system. The largest osteosclerotic changes were seen in the bones of the pelvis and in the vertebrae of the lumbar spine.

As this adenocarcinoma was diagnosed in a non-smoker, the patient was then tested for EGFR gene mutation in exons $18,19,20$ and 21 , and the arrangement of the ALK and ROS-1 gene was also determined. Negative results of the molecular studies disqualified the patient from targeted treatment.

The patient reported severe pain in the sternum area as well as the left hip radiating to the left lower limb. The pain was described as being acute, paroxysmal, waking her from sleep, with paresthesias of an intensity score of 6 on the NRS (numerical rating scale) of up to 10 point exacerbation scores. The patient was taking oxycodone $-10 \mathrm{mg}$ as needed, and ketoprofen $100 \mathrm{mg} 1 \times$ daily. Physical examination confirmed bone pain with a neuropathic component. Due to the ineffectiveness of the pain treatment, regular oral administration of opioids was recommended, i.e. oxycodone $2 \times 20 \mathrm{mg}$ with paracetamol $2 \times 1 \mathrm{~g}$ daily. Additionally, the co-analgesic pregabalin was orally prescribed $75 \mathrm{mg}$ twice daily. A satisfactory analgesic effect in the area of primary pain was obtained. Nevertheless, the patient reported very strong breakthrough pain. This pain, on the NRS scale, reached the highest values (9-10), had an acute onset (2-3 minutes) and lasted for about 30 minutes. It appeared without any specific cause, up to 4 times daily. Due to the described nature of the pain, it was advised to use an adjuvant water-based formulation of fentanyl intranasally at $100 \mu \mathrm{g}$ per dose. This supplementation brought about very satisfactory pain relief within minutes. Systemic treatment was implemented: palliative chemotherapy with pemetrexed $500 \mathrm{mg} / \mathrm{m}^{2}$ i.v. and cisplatin $50 \mathrm{mg} / \mathrm{m}^{2}$ i.v. every 21 days. Palliative radiotherapy was used for the areas of major pain in the sternum and left hip, using 20 Gy electron and photon fields in 4 fractions respectively. After two weeks, the pain was significantly reduced in the irradiated areas. Episodes of breakthrough pain occurred much less frequently of up to twice daily maximum. Bisphosphonates (zoledronic acid) were also used to induce osteoclast inactivation.

\section{Case no 2}

A 22-year-old patient was admitted to the A\&E department of a Gdańsk hospital after falling of a bicycle, injuring her right ankle and left ribcage. During the physical examination, ecchymoses were found in the vicinity of the injuries. No significant abnormalities were observed by laboratory testing. Diagnostic imaging via X-ray (XR) of both the ankle joint and chest were performed, and no fractures were seen. In the chest XR, widening of the mediastinum was noted. Further diagnostics led to the diagnosis of Ewing's sarcoma/ /PNET atclinically advanced stage III. The patient was qualified for standard systemic treatment according to the VCD/ /IE scheme (doxorubicin, cyclophosphamide, vincristine/ /ifosfamide, etoposide). Before initiating chemotherapy, a left-sided ovarectomy was performed with the goal of cryopreserving the ovarian tissue.

After the second cycle, it was essential to transfuse packed red blood cells due to anemia. Diagnostic imaging after the fourth cycle showed partial regression of the tumor. Unfortunately, after the next cycle - the fifth VCD, a rapid progression was seen. Second-line chemotherapy was initiated (doxorubicin, cisplatin), however this proved unable to prevent the drastic spread of the tumor within the chest and mediastinum, along with metastasis to the bones; primarily osteolytic in nature. Laboratory testing confirmed hypercalcemia $-12 \mathrm{mg} / \mathrm{dL}$, as well as hypokalemia $-2.8 \mathrm{mmol} / \mathrm{L}$. Clinically, signs of superior vena cava syndrome were noted, along with severe nausea and vomiting, as well as diarrhea. The patient complained of pain in the right shoulder and around the sternum, rating an 8 on the NRS, with acute attacks of a dull pain at 10/10 NRS. Daily tramadol at $2 \times 100 \mathrm{mg}$ daily, plus paracetamol + codeine $(500 \mathrm{mg}+30 \mathrm{mg}$ ) $2 \times$ day did not relieve her pain. Due to the increased intensity of the primary pain, it was decided to discontinue the oral weak opioid analgesics. In exchange, titrated morphine was given subcutaneously. After establishing an adequate daily dose, conversion to a transdermally applied dose in the form of a fentanyl patch was given at $50 \mu \mathrm{g} / \mathrm{h}$. Non-steroidal anti-inflammatory analgesics were added - ketoprofen $100 \mathrm{mg} 2 \times \mathrm{d}$ as well as corticosteroids in the form of dexamethasone $4 \mathrm{mg} 3 \times \mathrm{d}$. Due to the hypercalcemia, bisphosphonate supplementation (zolendronic acid) was given. Improvement in pain 
relief from the primary location was obtained, however the spontaneous breakthrough pain (9-10 NRS) was not well controlled. Initially, morphine was additionally given orally in $20 \mathrm{mg}$ doses about every 4 hours. This did not relieve the breakthrough pain entirely, and the patient complained of increased side effects (nausea and vomiting, constipation). Oral morphine was discontinued and intranasal fentanyl in a water-based formulation was given at doses of $100 \mu \mathrm{g} /$ /dose, as and when required. This change in medication brought about the expected analgesic effect. The patient required about 2-4 additional applications of this rescue medication to achieve complete pain relief. Immediately after stabilization of both the patient's general status and laboratory parameters, the patient was qualified for palliative radiotherapy of the mediastinum due to superior vena cava syndrome. After completing radiation therapy, she still requires multiple analgesics to treat her pain. Due to the invasion of almost the entire skeletal system, palliative radiotherapy for pain relief was not performed.

\section{Conclusions}

In contrast to primary neoplasms of the bone, which are rare, secondary metastasis to the bones is diagnosed in $70 \%$ of patients. Bone pain is often the first symptom of a generalized disease. Metastasis to the bone also threatens the patient's health with pathological fractures or spinal cord compression syndrome, which may require palliative radiotherapy or surgical intervention.

Pharmacotherapy of bone pain is classically treated with the three-step ladder of analgesia [3]. First line therapy includes non-steroidal anti-inflammatory drugs as well as opioids. It is important to remember to give these medications at the proper time intervals, as well as not giving weak opioids together with strong opioids. Due to the common characteristic of neuropathic pain, co-analgesics are often recommended such as anti-epileptics or anti-depressants [4]. It has been confirmed that anti-osteolytic medications, such as bisphosphonates, also have an analgesic effect. They can also be used to lower the serum calcium concentration [5]. Other anti-osteolytic medications with a confirmed analgesic effect include human molecular anti-bodies (IgG2) targeted against RANK-L and preventing activation of this receptor (receptor activating nuclear factor NF- $\kappa$ B) on the surface of osteoclasts and their precursors. At present, there is no evidence regarding the superiority of denozumab when compared to bisphosphonates for delivering an analgesic effect. It is known that denozumab is more effective at preventing adverse bone occurrences. Its use is related to a decreased risk of kidney damage or acute phase reactions, a similar risk of osteonecrosis of the jaw, and an increased risk of hypercalcemia [6].

In certain situations, it is standard practice to use anti-edema drugs such as corticosteroids. For such cases it is essential to use the appropriate route of administration and to prevent possible side effects such as bleeding from the gastrointestinal tract, immunosuppression leading to an increased risk of infection, or an impaired glucose tolerance. Effective systemic palliative treatment decreases the intensity of symptoms related to malignancies. The most effective of these is palliative radiotherapy, which brings about a very satisfactory local analgesic effect in $85 \%$ of patients, and a complete resolution of pain symptoms in about $15-60 \%$ of patients [7]. The analgesic effect, brought about as a result of the destruction of radiation-sensitive macrophages and osteoclasts, is independent of the histopathological type of cancer [8]. Such treatments can be given in 1, 4 or 5 fractions. Besides traditional radiotherapy, when treating bone metastases, stereotactic radiotherapy (SABRT) can also be used. This method is mainly emplyed in oligometastatic disease (1 to 3 metastatic foci) as well as in cases of metastasis localized around organs easily damaged by ionizing radiation, such as the spinal cord. SABRT not only delivers a quick reduction in pain intensity and improvement in neurological function in spinal cord compression syndrome, but thanks to the dramatically decreased dose outside of the irradiated field, the risk of damage to the spinal cord is diminished and additionally, it spares the bone marrow [9]. SABRT obviously does not repair compression fractures nor does it stabilize the spine.

In cases of multiple metastases to the bones, radioisotopes can be used, like stront or samar $[10,11]$. This kind of therapy is of a similar analgesic efficacy as teleradiotherapy. In cases of invasion of the entire skeleton, radioisotope therapy or radiation of half of the body in a single fraction can be used. However, it is important to remember that each method can cause long-lasting myelosuppression which can make the use of chemotherapy impossible. It has been shown that using radium chloride in patients with castration resistant prostatic cancer, not only delivers an analgesic effect but also significantly prolongs the overall survival, drastically improving the patient's quality of life; such treatment not being available in Poland [12].

In cases with an increased risk of fracture, or the occurrence of a fracture, spinal cord compression syndrome may require orthopedic or neurosurgical intervention and care as well as rehabilitation. It is essential to work together as a multi-disciplinary team, together with providing individualized therapy.

Studies show that in Poland over $75 \%$ of patients with pain due to malignant neoplasms do not obtain satisfactory pain relief [13]. Proof of this can be seen in the 20-fold lower usage of morphine equivalents in $\mathrm{kg}$ per capita in Poland when compared to countries with a similar population such as Canada.

Problems related to pain management are still a relevant issue and require further refinement in the education of 
both medical students and physicians, independent of their specialization(s).

\section{Conflict of interest: none declared}

\section{Prof. Renata Zaucha, MD, PhD}

Department of Clinical Oncology and Radiotherapy

Medical University of Gdańsk

7 Debinki St., 80-211 Gdańsk, Poland

e-mail:rzaucha@gumed.edu.pl

Received: 4 Aug 2016

Accepted: 15 Mar 2017

\section{References}

1. Zalecenia postępowania diagnostyczno-terapeutycznego wnowotworach złośliwych 2013 rok. T. 1. Krzakowski M, Warzocha K (ed.). Gdańsk: Via Medica, 2013.

2. Stany nagłe w onkologii - wystepowanie i leczenie. T. II. Szawłowski A Wallner G (ed.). Poznań: Termedia Wydawnictwa Medyczne, 2015.

3. WHO. Cancer pain relief: with a guide to opioid availability. 2nd ed. Geneva: World Health Organization, 1996.

4. Saarto T, Wiffen P. Antidepressants for neuropathic pain (Cochrane Review). Cochrane Database Syst Rev 2007; (4): CD005454.

5. Wong R, Wiffen PJ. Bisphosphonates for the relief of pain secondary to bone metastases. Cochrane Database Syst Rev 2002; (2): CD002068.
6. Sivolella S, Lumachi F, Stellini E et al. Denosumab and anti-angiogenetic drug-related osteonecrosis of the jaw: an uncommon but potentially severe disease. Anticancer Res 2013; 33: 1793-1797.

7. van der Velden JM, Verkooijen HM, Seravalli E et al. Comparing conventional radiotherapy with stereotactic body radiotherapy in patients with spinal metastases: study protocol for an randomized controlled trial following the cohort multiple randomized controlled trial design. BMC Cancer 2016; 16: 909.

8. Yamada Y, Katsoulakis E, Laufer I et al. The impact of histology and delivered dose on local control of spinal metastases treated with stereotactic radiosurgery. Neurosurg Focus 2017; 42: E6. doi: 10.3171/2016.9. FOCUS16369.

9. Schipani S, Wen W, Jin JY et al. Spine radiosurgery: A dosimetric analysis in 124 patients who received 18Gy. Int J Radiat Oncol Biol Phys 2012; 84: e571-576.

10. Oosterhof GO, Roberts JT, de Reijke TM et al. Strontium(89) chloride versus palliative local field radiotherapy in patients with hormonal escaped prostate cancer: a phase III study of the European Organisation for Research and Treatment of Cancer, Genitourinary Group. Eur Urol 2003; 44: 519-526.

11. Roqué I Figuls $M$, Martinez-Zapata MJ, Scott-Brown M et al. Radioisotopes for metastatic bone pain. Cochrane Database Syst Rev 2011; (7): CD003347.

12. Parker $C$, Nilsson $S$, Heinrich $D$ et al. Alpha emitter radium-223 and survival in metastatic prostate cancer. NEngl J Med 2013; 369: 213-223.

13. Deandrea S, Montanari M, Moja L et al. Prevalence of undertreatment in cancer pain. A review of published literature. Ann Oncol 2008; 19: 1985-1991.

14. www.treatthepain.org 